\title{
The Influence of the form Error after Rotor Manufacturing on the Output Characteristics of an Orbital Hydraulic Motor
}

\author{
Anatolii Panchenko ${ }^{1}$, Angela Voloshina ${ }^{1}$, Irina Milaeva ${ }^{1}$, Igor Panchenko $^{1}$, Olena Titova ${ }^{2}$ \\ ${ }^{1}$ Tavria State Agrotechnological University \\ ${ }^{2}$ Melitopol State Pedagogical University \\ *E-mail: voloshinaa2012@gmail.com
}

\begin{abstract}
The technology of rotors manufacturing for orbital hydraulic motors causes a certain form error of the gear surface. This leads to oscillations of the diametrical gap between the rotors. Such oscillations have a negative effect on the change in the output characteristics of the orbital motor. The previous studies on the issue consider the interaction between the rotors of orbital hydraulic machines as a gapless (theoretical) connection. The authors do not take into account that between the rotors of the orbital hydraulic motor, there is no rigid kinematic connection. This allows the internal rotor to move randomly within the diametrical gap (self-aligning). The study led to the development of the mathematical models that describe the relationship between the design features of the rotors and the output characteristics of an orbital hydraulic motor. To simulate the oscillation of the diametrical gap, the limiting deviations for the form error of the rotor gear surfaces have been substantiated. Investigations revealed that fluctuations in the form error of the rotor gear surface have a significant effect on the variation of the diametrical gap. The limiting deviations in the form errors of the gear surface manufacturing enabled to eliminate the oscillation of the diametrical gap and to ensure stability of the output characteristics for the orbital hydraulic motor.
\end{abstract}

Keywords: form error after rotor manufacturing;orbital hydraulic motor.

\section{Introduction}

Current trends in hydraulic drive application for self-propelled machinery design require development of new and improvement of existing designs of orbital hydraulic machines as well as new approaches to improvement of their output characteristics. The use of orbital hydraulic motors in the drives of active working tools for self-propelled machinery makes great demands on changing the output characteristics of these hydraulic motors. It is possible to do it at the design stage $[1,3]$.

Technological difficulties, which occur during manufacture of the rotors of an orbital hydraulic motor, are caused by their design features. The difficulties provide a certain error in the shape of the gears surface of the rotors (Figure 1). It leads to oscillations of the diametrical gap between the outer (Figure 1, a) and inner (Figure 1, b) rotors [4-8]. During operation, oscillations of the gap have a negative effect on the changes in the output characteristics of an orbital hydraulic motor.

Thus, an actual connection of the rotors in the orbital motor differs from the theoretical one, as there is a diametrical gap and there is no rigid kinematic connection between the internal and external rotors [9]. Therefore, the investigation of the influence, which a diametrical gap between internal and external rotors brings to the change of hydraulic motor output characteristics, is an actual problem. Solution of the problem will allow to provide necessary output parameters of hydraulic motors at the design stage.

\subsection{Analysis of Recent Studies and Publications}

Currently, much attention is paid to the design of rotors for gerotor pumps [4]. A mathematical model, which describes the change in the loads of gearing, has been developed. Experimental studies for prototypes of gerotor pumps have been carried out. The issues of the kinematic connection between the rotors were not considered.

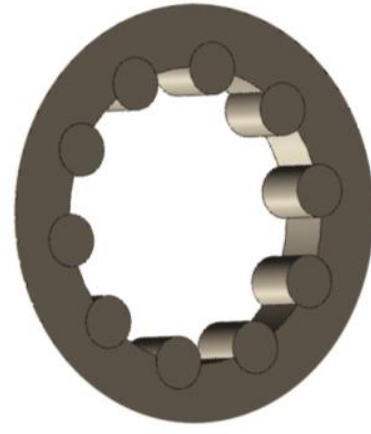

a

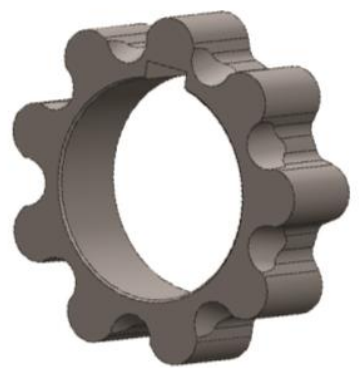

$\mathrm{b}$
Fig. 1: The outer (a) and inner (b) rotors of orbital hydraulic motors.

The forces which act in the gearing of the gerotor pump [5] are considered. The models that take into account the compression state of the power fluid have been prepared. We developed some recommendations for the design of a gerotor for hydraulic machines as well. The relationship between the design features of the rotors and the output characteristics of hydraulic machines has not been studied. The issues associated with wear of the rotors working surfaces in orbital hydraulic motors [6] were investigated. The model which describes the tribological changes in the geometry of the working surfaces of the rotors in those hydraulic motors has been developed. However, the influence of the form 
error the after the rotor manufacturing on the output characteristics of the orbital hydraulic motor was not investigated. We proposed a program for designing of hypocycloidal surfaces of rotors for gerotor machines [7]. It takes into account the equations of hydrodynamics or fluid flow in working chambers. The limiting deviations in the form of manufacturing of the gearing surfaces of the inner and outer rotors in the orbital hydraulic motor were not substantiated.

The recent studies on displacement kinematics, power interaction and the design of internal and external rotors of orbital hydraulic machines [3-8] show one of the main limitations. The fact is that the interaction between rotors was considered as a theoretical (gapless) connection where there are no form errors of the rotor gear surfaces. These works did not take into account that there is no rigid kinematic connection between the rotors in the orbital hydraulic motor. This allows the internal rotor to move arbitrarily within the diametric gap and perform self-aligning. In this case, the rotor can assume various interrelations which depend on the error in the shape of its gear profiles. Thus, the actual coupling differs from the theoretical one as it has a diametrical gap.

\subsection{Statement of the Objective and Tasks Of The Study}

The objective of the study was to improve the output characteristics of an orbital hydraulic motor by restriction of limiting deviations of the error in the shape of rotor gear surfaces. To achieve the assigned objective the following tasks have been set:

- determine a relationship between structural features of the rotors and output characteristics of an orbital hydraulic motor;

- substantiate limiting deviations which are acceptable for the form error of gear surfaces of internal and external rotors in an orbital hydraulic motor;

- investigate how the form error after the rotor manufacturing influences the output parameters of the orbital hydraulic motor.

\section{The Basic Part of the Study}

The main parameters which describe the output characteristics of a hydraulic motor are the torque $M_{t o r}$, developed by the motor, and the corresponding rotation frequency $n$.

Theoretical (calculated) torque on the shaft of the orbital hydraulic motor (Figure 2) is expressed by the dependence $[3,10]$ :

$$
M_{\text {tor }}=2 e \cdot \Delta p \cdot b \cdot R_{1} \cdot\left(z_{1}+1\right),
$$

where e is the center-to-center distance between inner and outer rotors, $\mathrm{mm} ; \Delta \mathrm{p}$ is pressure drop of hydraulic fluid in the motor, $\mathrm{MPa}$; $b$ is the rotor width, $\mathrm{mm} ; \mathrm{R}_{1}$ is the radius of a dividing circle which defines the location of the teeth centers for the inner rotor, $\mathrm{mm} ; \mathrm{z}_{1}-$ the number of the inner rotor teeth.

The expression (1) can also be represented (according to [9]) as

$M_{\text {tor }}=F \cdot h$,

where $\mathrm{F}$ is the resultant force from the action of power fluid pressure; $h$ is the application arm of the resultant force $F$, it is defined by the radius $R_{1}$.

$$
F=2 e \cdot \Delta p \cdot b \cdot\left(z_{1}+1\right) .
$$

Figure 2 shows that the value of the arm $h$ of the resultant force $F$ is defined as

$$
h=O_{1} O_{2}+O_{1} O_{3}+O_{3} O_{4} \text {, }
$$

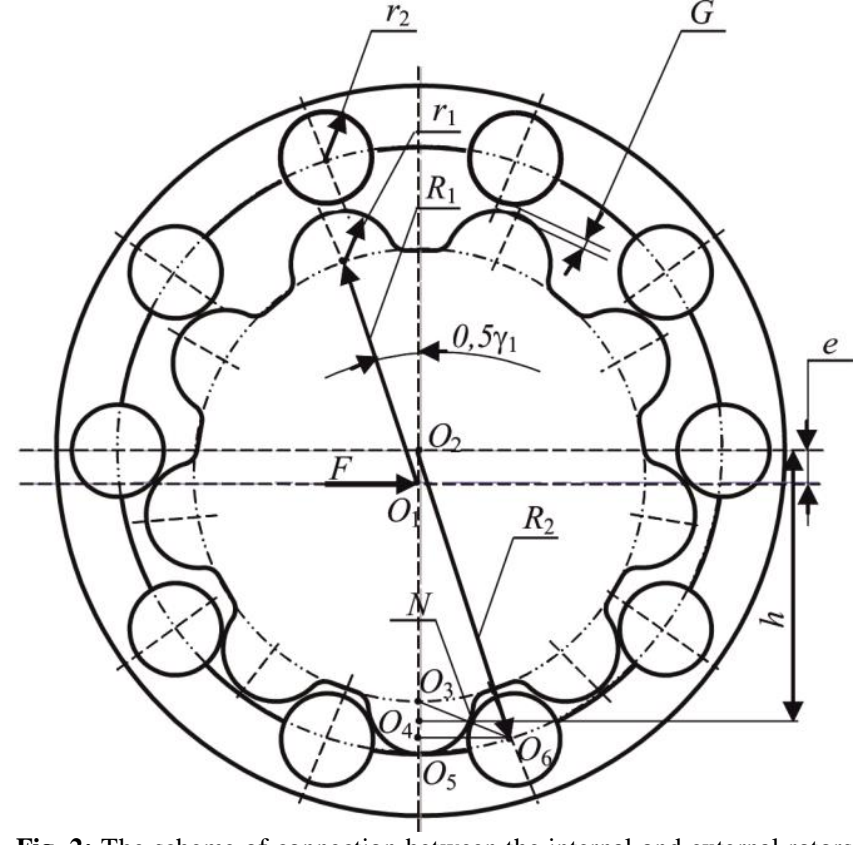

Fig. 2: The scheme of connection between the internal and external rotors of an orbital motor: $O_{1}$ is the center of the inner rotor; $O_{2}$ is the center of the outer rotor; $O_{3}$ is the center of the inner rotor gearing; $O_{6}$ is the center of the outer rotor gearing; $R_{1}$ is the radius of a dividing circle which defines the location of the gearing centers in the inner rotor; $R_{2}$ is the radius of a dividing circle which defines the location of the gearing centers in the outer rotor; $r_{1}$ is the radius of the inner rotor gearing; $r_{2}$ is the radius of the outer rotor gearing; $e$ is center-to-center distance between the inner and outer rotors; $F$ is the resultant force from the action of power fluid pressure; $\mathrm{h}$ is the application arm of the $F$ resultant force; $G$ is the diametrical gap.

where $\quad O_{1} O_{2}=e ; \quad O_{1} O_{3}=R_{1} ; \quad O_{3} O_{4}=O_{3} N \cdot \cos \alpha ; \quad O_{3} N=r_{1}$ $\cos \alpha=\frac{O_{5} O_{6}}{O_{3} O_{6}} ; O_{5} O_{6}=O_{2} O_{6} \cdot \sin \left(0,5 \gamma_{2}\right) ; O_{3} O_{6}=r_{1}+r_{2} ;$

$\mathrm{O}_{2} \mathrm{O}_{6}=R_{2} ; \quad 0,5 \gamma_{2}=\pi / z_{2}$.

Taking into account expressions (5), after appropriate substitutions and transformations, expression (4) takes the form:

$h=e+R_{1}+r_{1} \cdot \frac{R_{2} \cdot \sin \left(\pi / z_{2}\right)}{r_{1}+r_{2}}$,

where $R_{2}$ is the radius of a dividing circle which defines the location of the teeth centers in the outer rotor, $\mathrm{mm} ; \mathrm{z}_{2}$ is the number of the outer rotor teeth; $r_{1}$ is the radius of the inner rotor teeth, $\mathrm{mm} ; \mathrm{r}_{2}$ is the radius of the outer rotor teeth, $\mathrm{mm}$.

Kinematic analysis of the displacement of internal and external rotors has shown $[3,9]$ that the shape of the rotors gear contour $E$ has a significant effect on the value of the diametrical gap $G$ (Figure 2). The error in the form of the gear contour $E$ is a complex indicator and depends on the relationship between geometric parameters $E=f\left(R_{1}, r_{1}, \gamma_{1}, R_{2}, r_{2}, \gamma_{2}\right)$.

The initial value of the diametric gap is determined by the sum of its technological (specified by the drawing) gap $G_{t}$ as well as the deviations caused by the form error $E$ of the internal and external rotors.

$G=G_{t} \pm E$,

where ' + ' or ' - ' sign for the E component is determined by specific deviations in the form error of inner and outer rotors.

Considering expressions (6) and (7), the variation of $h$, which 
depends on the contact in the $i^{\text {th }}$ pair of teeth, can be expressed in terms of the geometrical parameters for the internal rotor, while the diametrical gap $G$ is varying over its whole range:

$h_{i}=h \cdot \cos \frac{2 \pi}{Z_{1}}(i-1) \pm \frac{\left(G_{t} \pm E\right)}{2}$,

where $\mathrm{i}$ is the ordinal number of the internal rotor tooth which determines its instantaneous rotation center (considering $\mathrm{E}$ ), $\mathrm{i}=1$, $2, \ldots, \mathrm{z}_{1}$.

When the diametrical gap is less than the critical value $G_{c r}$, the instantaneous rotation center of the gear always passes through the conditional tooth No. $1(i=1)$. Consequently, $\cos \left(2 \pi / z_{1}\right) \cdot(i-1)=1$ and expression (8) takes the form:

$$
h_{1}=h \pm \frac{\left(G_{t} \pm E\right)}{2} .
$$

Then, taking into account expression (9), the equation (6) takes the form:

$$
h_{1}=e+R_{1}+r_{1} \cdot \frac{R_{2} \cdot \sin \frac{\pi}{z_{2}}}{r_{1}+r_{2}} \pm \frac{\left(G_{t} \pm E\right)}{2} .
$$

When the values of the resultant force $F$ are substituted into (2), the change in the center-to-center distance $e$ by the gap variation \pm $G / 2$ and the expression (7) are taken into account, we obtain the dependence for determining the torque:

$$
M_{\text {tor }}=2\left(e \pm \frac{\left(G_{t} \pm E\right)}{2}\right) \cdot \Delta p \cdot b \cdot\left(z_{1}+1\right) \cdot h_{i},
$$

where ' + ' or ' - ' sign for the $\left(\mathrm{G}_{t} \pm \mathrm{E}\right) / 2$ component is determined by the position of the inner rotor.

Kinematics of the rotors displacement was analyzed as well. It showed that during operation, when the value of a diametrical gap changes from $G$ to $G_{c r}$, the torque varies quite significantly. These oscillations are caused by the changes in geometric parameters of internal and external rotors. The changes of geometric parameters, which significantly influence the $h$ value [9], appear during manufacture of the rotors.

When during operation the values of the diametrical gap exceed the critical value $G_{c r}$, the instantaneous rotation center of the gear passes through the conditional tooth No. 2, No. 3, etc. The value of $h$ is determined by the expression (6), therefore the torque sharply decreases and becomes $\cos \left(2 \pi / z_{1}\right) \cdot(i-1)$ times less. Analysis of the mathematical dependencies, which describe the change in the torque of the orbital motor after the variation in the diametrical gap, enables to explain the situation. When the diametrical gap variation is in the $G \ldots G_{c r}$ range, the change in torque is described by a pulsating curve with a constant average value. If the range of values is $G>G_{c r}$, the torque will decrease sharply and, consequently, will be described by a parabolic dependence.

As the result of theoretical studies, a mathematical apparatus has been developed. That makes it possible to describe the relationship between the design features of internal and external rotors and the output characteristics of an orbital hydraulic motor.

\section{Results and Discussion}

For the purposes of our study, a mathematical apparatus has been built. It was realized on the Vissim visual modeling package. It enabled to investigate the influence of a diametrical gap on the output characteristics of a planetary motor. The variation in the form error of the internal and external rotor gears have been modeled and considered as well.

To determine the influence of the diametrical gap oscillations on the change in output parameters of orbital motors, we measured the deviations of the form error which occurs during manufacturing [9]. The form error after rotor manufacturing was checked according to the methods given in [11]. For the upgraded hydraulic motor, the deviation of the error in the shape of the gear surface was set considering capabilities of the production technology [9].

The form error of the rotors has been taken into account while the diametrical gap variation was being modeled. The Vissim package was used to model both production and upgraded hydraulic motors diametrical gaps.

To simulate the operation of the rotors of an orbital hydraulic motor, we took the following initial data and starting conditions:

- working volume of the hydraulic motor is $V=160 \mathrm{~cm}^{3}$;

- angular velocity of the hydraulic motor shaft is $\omega=68 \mathrm{~s}^{-1}$;

- theoretical hydromechanical efficiency, which was calculated, equals to $\eta_{t . h m}=0,95$.

Figures 3-7 show the structural and functional diagram of the interaction between the external and internal rotors. It allows to model their operation.

The initial data (working volume, angular velocity and hydromechanical efficiency) of the hydraulic motor were set by block $\mathrm{A}$ (Figure 3 ).

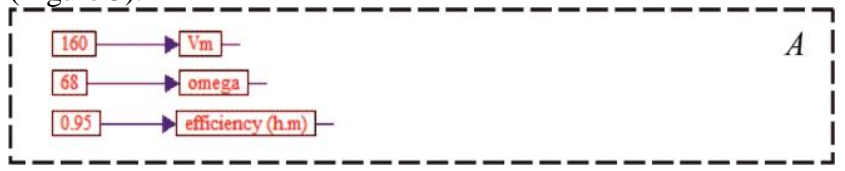

Fig. 3: The initial data block.

$\boldsymbol{B}$ block (Fig. 4) allows to set the rotation of the motor shaft by modeling the displacement of the internal rotor

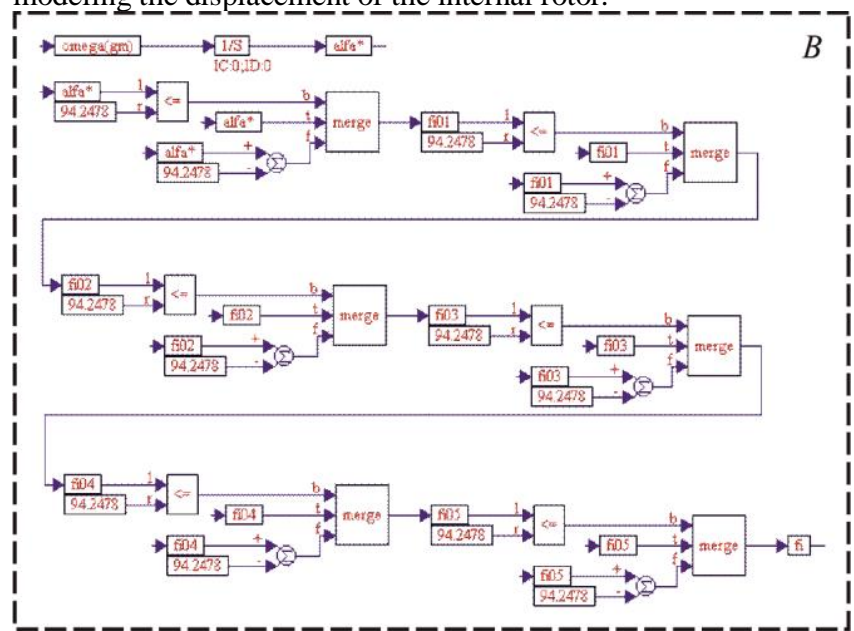

Fig. 4: The block for modeling of the hydraulic motor shaft rotation.

The form error after the manufacture of the external and internal rotors and the technological clearance are set by the $\boldsymbol{C}$ block (Figure 5)

D block (Figure 6) allows to determine the change in the diametrical gap between the outer and inner rotors at any moment of time.

$\boldsymbol{F}$ block (Figure 7) makes it possible to set the hydromechanical losses when the error in the form after the rotors manufacturing as well as the technological clearance are taken into account.

The simulation results showed (Figure 8) that during the orbital motor operation, constant variation (oscillation) of the diametrical gap occurs, as there is no rigid kinematic connection between its rotors. For a standard hydraulic motor, these oscillations are 


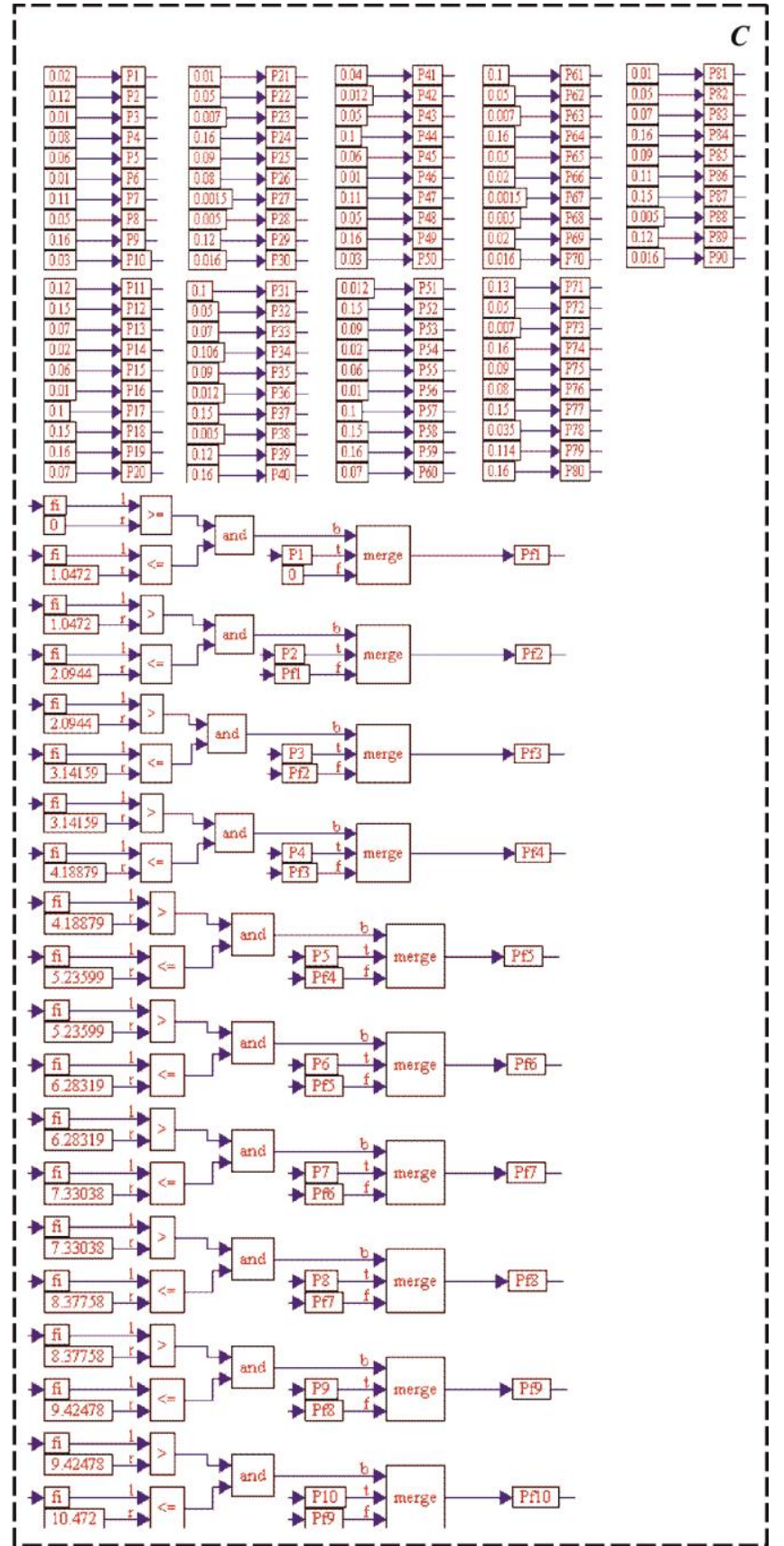

Fig. 5 . The block for modeling of the form error after the manufacture the external and internal rotors and the technological clearance as well.

$G=0.055$ to $0.21 \mathrm{~mm}$ (curve 1 ), and for the upgraded one the range is $G=0.05$ to $0.06 \mathrm{~mm}$ (curve 2 ).

The oscillations of the diametrical gap have chaotic and cyclic character at each new position of the rotor teeth and the gear. It repeats after a certain cycle $z_{c}=z_{1} \cdot z_{2}$.

Significant difference in the oscillation amplitude of the diametrical gap $G$ (Figure 8) for production and upgraded hydraulic motors is explained by the large total form error after the rotor manufacturing $E=0.15 \mathrm{~mm}$ for the production hydraulic motor. The upgraded hydraulic motor has the form error after the rotor manufacturing of $E=0.01 \mathrm{~mm}$.

The change in the hydromechanical efficiency of a displacement system was modeled as well. The results of modeling show (Figure 9) that hydromechanical losses have chaotic character too. It is inversely proportional to the variation of the diametrical gap. The relative position of the rotor and the gear influences the deviations of the hydromechanical efficiency. The values make up $\eta_{h m}=0.8$ to 0.94 (for the production hydraulic motor; curve 1), and $\eta_{h m}=0.93$ to 0.95 (for the upgraded hydraulic motor; curve 2).

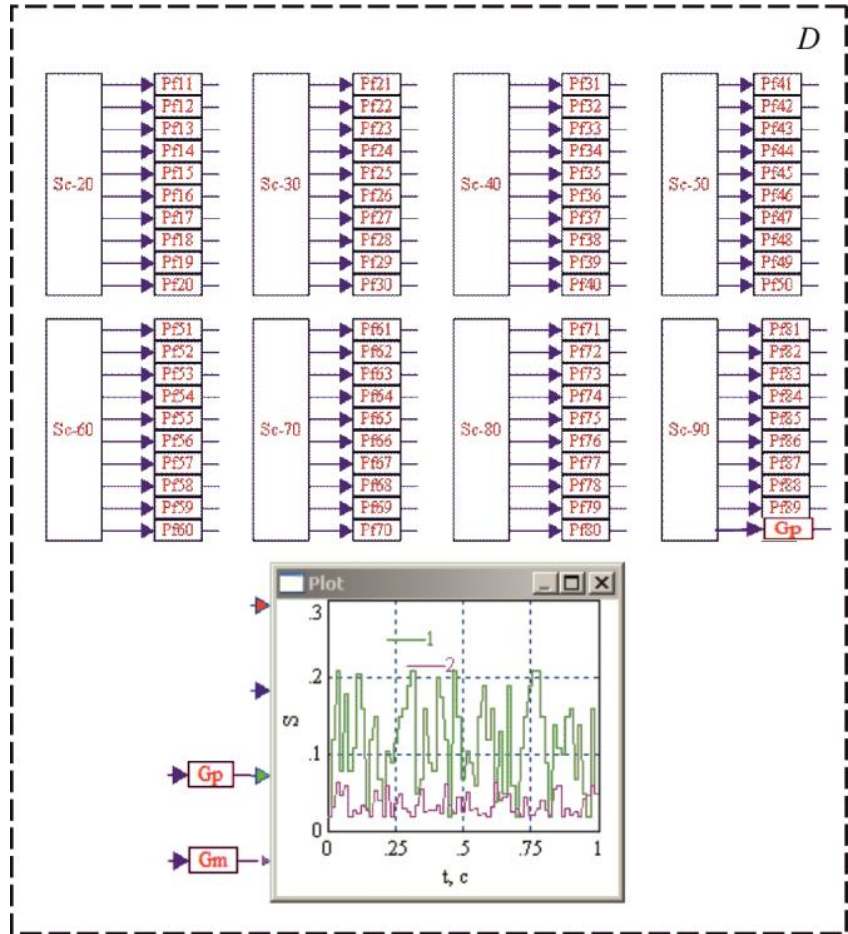

Fig. 6: The block for modeling of the change in the diametrical gap between the outer and inner rotors at any moment of time.

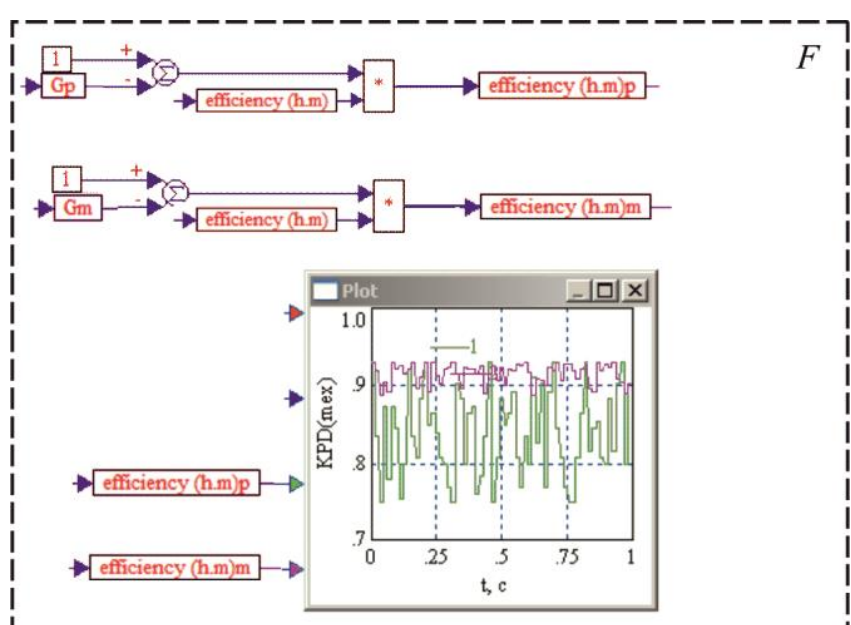

Fig. $\overline{7}$ : The block for modeling of the change in the hydraulic and mechanical losses when the error in the form after the rotors manufacturing as well as the technological clearance are taken into account.

Oscillations of hydromechanical efficiency repeat after $z_{c}=z_{1} \cdot z_{2}$ cycles.

Changes in the diametrical gap and hydromechanical efficiency cause significant fluctuations in the torque (Figure 10). Moreover, the amplitude of the torque oscillations for the production hydraulic motor is $60 \mathrm{~N} \cdot \mathrm{m}$. It ranges $M_{t o r}=325$ to $385 \mathrm{~N} \cdot \mathrm{m}$ (curve 1), and for the upgraded motor $M_{\text {tor }}=382$ to $388 \mathrm{~N} \cdot \mathrm{m}$ (curve 2). Significant changes (oscillations) of the torque for the production hydraulic motor are caused by oscillations of the diametrical gap. As a consequence, hydromechanical efficiency changes as well. The research of the variation dynamics for the diametrical gap and the hydromechanical efficiency of the orbital hydraulic motor have established that:

- the existing technology of the internal and external rotors manufacturing does not provide given geometry of their tooth profile (the form error of the rotor manufacture is $E=0.15 \mathrm{~mm}$ ); - oscillations in the form error of the rotors are chaotically cyclic in nature, repeated after $z_{c}=z_{1} \cdot z_{2}$ cycles and have a significant effect on the variation of the diametrical gap; 


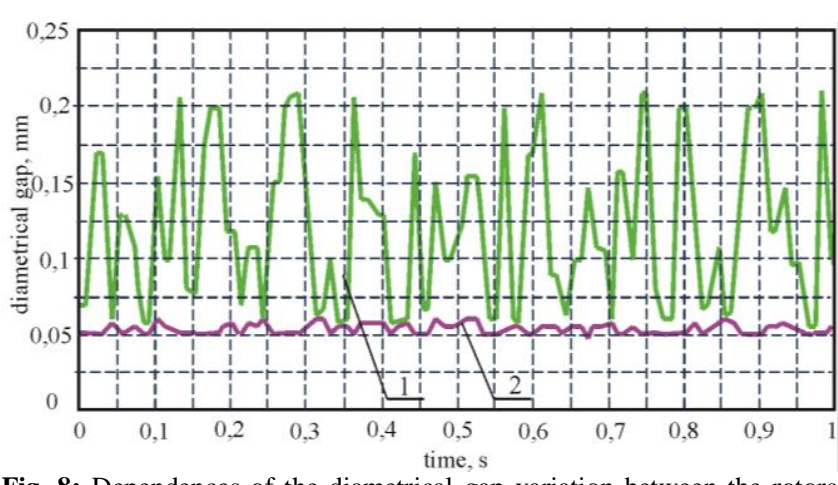

Fig. 8: Dependences of the diametrical gap variation between the rotor during operation: 1 - production hydraulic motor; 2 - upgraded hydraulic motor.

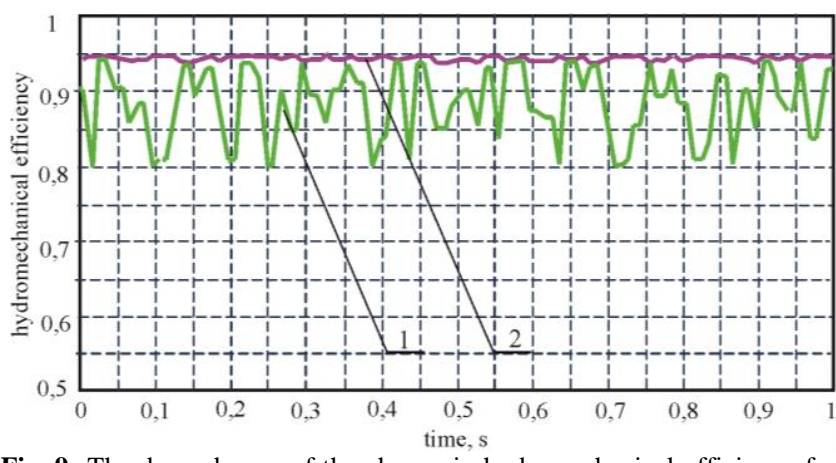

Fig. 9: The dependences of the change in hydromechanical efficiency for the orbital hydraulic motor: 1 - production motor; 2 - upgraded motor.

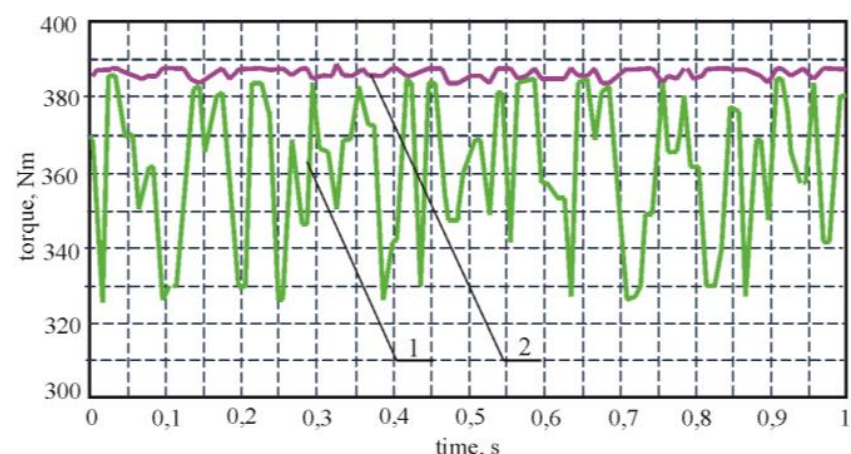

Fig: 10. The dependences of the torque variation for the orbital hydraulic motor: 1 - production motor; 2 - upgraded motor.

- the oscillations of the hydromechanical efficiency are inversely proportional to the oscillations of the diametrical gap and make up $\eta_{h m}=0.8$ to 0.94 (for the production hydraulic motor), and $\eta_{h m}=$ 0.93 to 0.95 (for the upgraded one);

- the oscillations of the torque have the same character as the changes in the hydromechanical efficiency and are caused by the instability in its values. The torque oscillations for the series hydraulic motor are $M_{\text {tor }}=325$ to $385 \mathrm{~N} \cdot \mathrm{m}$, but for the upgraded one they are $M_{t o r}=382$ to $388 \mathrm{~N} \cdot \mathrm{m}$.

\section{Conclusion}

The effect of design features of the rotors on the output characteristics of an orbital hydraulic motor were studied using a special mathematical apparatus which has been developed to describe those relationships. Limiting deviations of the form error in the toothed surfaces of the internal and external rotors were substantiated to simulate the variation (oscillation) of the diametrical gap.

The form error which occurs after the rotor manufacturing influences significantly the output characteristics of the orbital hydraulic motor. In fact it was found that the variations in the form error of the internal and external rotors are of chaotical cyclical nature and have a dramatic effect on the variation of the diametrical gap. Fluctuations in the hydromechanical efficiency of the orbital hydraulic motor were 0.8 to 0.94 (for the production motor) and 0.93 to 0.95 (for the upgraded one). Torque fluctuations are similar to the changes in hydromechanical efficiency and are caused by instability in its values. The torque oscillations were 325 to $385 \mathrm{~N} \cdot \mathrm{m}$ (for the production hydraulic motor) and 382 to $388 \mathrm{~N} \cdot \mathrm{m}$ (for the upgraded one).

\section{References}

[1] Panchenko A. I. "Hydraulic machines for driving active working bodies and chassis of the mobile agricultural machinery", Machinery in the agroindustrial complex, No.3, (2006), pp.11-13

[2] Panchenko A. I., Voloshina A. A., Panchenko I. A., "Development of planetary hydraulic motors for the power hydraulic drives of mobile machinery", MOTROL, Vol.17, No.9, (2015), pp.29-36

[3] Panchenko A., Voloshina A., Boltyansky O., and others, "Designing the flow-through parts of distribution systems for the PRG series planetary hydraulic motors", Eastern-European Journal of Enterprise Technologies, Vol. 3, No.1 (93), (2018), pp.67-77, doi: 10.15587/1729-4061.2018.132504

[4] Stryczek J., Bednarczyk S., Biernacki K., "Strength analysis of the polyoxymethylene cycloidal gears of the gerotor pump", Archives of Civil and Mechanical Engineering, Vol.14, No.4, (2014), pp.647-660, doi: 10.1016/j.acme.2013.12.005

[5] Chang Y. J., Kim J. H., Jeon C. H., Kim Chul, Jung S. Y., "Development of an Integrated System for the Automated Design of a Gerotor Oil Pump", Journal of Mechanical Design, Vol.129, No.10, (2006), pp.1099-1105, doi: 10.1115/1.2757629

[6] Choi T. H., Kim M. S., Lee G. S., Jung S. Y., Bae J. H., Kim C., "Design of Rotor for Internal Gear Pump Using Cycloid and Circular-Arc Curves", Journal of Mechanical Design, Vol.134, No.1, (2012), 011005-12, doi:10.1115/1.4004423

[7] Stryczek J., Bednarczyk S., Biernacki K., "Gerotor pump with POM gears: Design, production technology, research", Archives of Civil and Mechanical Engineerin, Vol.14, No.3, (2014), pp.391397, doi: 10.1016/j.acme.2013.12.008

[8] Biryukov B. N., Rotary piston hydraulic machines, Mechanical engineering, (1977)

[9] Panchenko A. I., Voloshina A. A., Planetary rotary hydraulic motors. Calculation and designing: monograph, Publishing and Printing Center "Lux", (2016), 236 p.

[10] Erasov F.N., New planetary hydraulic drive machines, Kiev, (1969)

[11] Panchenko A. I., Voloshina A. A., Guiva S. D., Leus G., Methodology of accuracy examining for manufacturing of displacing and distribution systems elements in planetary hydraulic machines, Proceedings of the Tavria State Agrotechnological University, Vol.16, No.2, (2016), pp.3-27 